\title{
Autonomous and non-autonomous traits mediate social cooperation in Dictyostelium discoideum
}

\author{
Nameeta Mujumdar ${ }^{1, \dagger}$, Ashvini Kumar Dubey $^{2}$, Krithi Nandimath $^{3}$ \\ and VidYanAND NanJundiah ${ }^{1,2,3, *}$ \\ ${ }^{1}$ Department of Molecular Reproduction, Development and Genetics, ${ }^{2}$ Centre for Ecological Sciences, Indian \\ Institute of Science, Bangalore 560 012, India \\ ${ }^{3}$ Jawaharlal Nehru Centre for Advanced Scientific Research, Bangalore 560012, India \\ ${ }^{\dagger}$ Present address: Division of Basic and Translational Research, University of Minnesota, Minneapolis, \\ MN 55455, USA \\ *Corresponding author (Fax, +91-80-23600999; Email, vidya@ces.iisc.ernet.in)
}

In the trishanku (triA ${ }^{-}$) mutant of the social amoeba Dictyostelium discoideum, aggregates are smaller than usual and the spore mass is located mid-way up the stalk, not at the apex. We have monitored aggregate territory size, spore allocation and fruiting body morphology in chimaeric groups of (quasi-wild-type) Ax2 and triA cells. Developmental canalisation breaks down in chimaeras and leads to an increase in phenotypic variation. A minority of triA ${ }^{-}$cells causes largely Ax2 aggregation streams to break up; the effect is not due to the counting factor. Most chimaeric fruiting bodies resemble those of Ax 2 or trit ${ }^{-}$. Others are double-deckers with a single stalk and two spore masses, one each at the terminus and midway along the stalk. The relative number of spores belonging to the two genotypes depends both on the mixing ratio and on the fruiting body morphology. In double-deckers formed from 1:1 chimaeras, the upper spore mass has more Ax 2 spores, and the lower spore mass more triA ${ }^{-}$spores, than expected. Thus, the traits under study depend partly on the cells' own genotype and partly on the phenotypes, and so genotypes, of other cells: they are both autonomous and non-autonomous. These findings strengthen the parallels between multicellular development and behaviour in social groups. Besides that, they reinforce the point that a trait can be associated with a genotype only in a specified context.

[Mujumdar N, Dubey AK, Nandimath K and Nanjundiah V 2011 Autonomous and non-autonomous traits mediate social cooperation in Dictyostelium discoideum. J. Biosci. 36 505-516] DOI 10.1007/s12038-011-9084-3

Development...is in essence the interaction between parts... But if the individual merges into an association of individuals, then there will be an extension of the principle of development to include these larger associations, for here also the colony of the society is integrated and unified by the interaction between parts. If in evolution there has been... an increase in the compounding of living units (into colonies, multicellular organisms and societies) then there must also have been a corresponding extension of development. (Bonner 1958)

\section{Introduction}

The extraordinary reliability of multicellular development under standard environmental conditions gives the impression that its course is pre-programmed, meaning irrevocably specified, in the genome. However, quite apart from the effect of the external environment, development involves reciprocal interactions between different cells. This means that the phenotype of a developing cell must depend not only on its own genotype but also on the phenotypes, and therefore the genotypes, of other cells. The last inference is meaningless in the case normal metazoan development: being clonal deriv-

Keywords. Aggregation; canalisation; development; phenotype interactions; phenotypic plasticity; quorum sensing; social selection; trishanku

Abbreviations used: CF, counting factor; CM, conditioned medium; CSM, cellular slime mould; DD, double-decker; US, upper sorus; LS, lower sorus 
atives of the zygote, the cells of an embryo are genetically identical. Genetic mosaicism is the occurrence of spatially restricted mutant cells in an otherwise wild-type embryo (or vice versa). Spontaneous or induced mosaics are a powerful tool for identifying genes whose activities mediate intercellular interactions (Stern 1968). Another option is to monitor development in chimaeras, namely, in embryos that are constituted by combining cells of different genotypes (Mintz and Silvers 1967; Le Douarin and Teillet 1974). Chimaeras and mosaics are especially useful when the phenotypes associated with each genotype are qualitatively different, because then one can investigate the extent to which the phenotype of a cell or tissue can be influenced by phenotypes other than its own, i.e. to address the issue of autonomy versus non-autonomy (Stern and Tokunaga 1967; Morata and Lawrence 1977; Babu and Bhat 1986). Autonomy means that the phenotype of a cell depends on its genotype; nonautonomy is the hallmark of regulative development (Gilbert 2000), of which the cellular slime mould (CSM) Dictyostelium discoideum is an exemplar (Raper 1940).

The present study involves chimaeras in D. discoideum. CSMs live in soil and animal dung; their life cycle consists of alternating free living and social phases (Bonner 1967; Raper 1984; Kessin 1997). Starved amoebae attract each other over distances $\sim 1 \mathrm{~mm}$ or more via a chemical signal and form compact aggregates that can be compared to multicellular embryos. The completed aggregate forms a motile quasi-cylindrical structure, the slug, which transforms itself into an erect fruiting body consisting of a spherical mass of starvation-resistant spores held aloft by a columnar stalk of dead amoebae. Following possible passive dispersal and under favourable conditions, an amoeba emerges from a spore, feeds on soil bacteria and divides by mitosis until starvation sets in and triggers the next social phase. Natural CSM aggregations can contain more than one clone (Fortunato et al. 2003; Gilbert et al. 2009; Sathe et al. 2010), although most laboratory studies of development involve clonal cultures.

The trishanku (triA) gene of $D$. discoideum has pleiotropic effects on development (Jaiswal et al. 2006; Mujumdar et al. 2009). The loss of function trit $A^{-}$mutant shows two striking differences from the wild type: aggregation streams break up after some hours and the sorus (spore mass) stops midway as it ascends along the stalk. We have monitored these two traits - integrity versus break-up of streams and normal versus abnormal positioning of the spore mass - in $\mathrm{Ax} 2+$ triA $^{-}$chimaeras. The aim was to see whether they could be attributed exclusively to autonomous or non-autonomous properties of cells. It turns out that they cannot. Our study illustrates the advantage of using genetically heterogeneous social groups for uncovering the complex interactions that underlie social behaviour (Nanjundiah and Sathe 2011).

\section{Materials and methods}

\subsection{Reagents and media}

Protease peptone, yeast extract and BactoAgar were obtained from Difco Laboratories, USA, and other reagents from Ranabaxy, India, or Himedia, India. Antibiotics were from Amersham Pharmacia Biotech, UK, or Sigma Chemical Company, USA. The standard nonnutrient medium used for starving cells and preparing agar plates was potassium phosphate buffer ('KK2'; $16 \mathrm{mM} \mathrm{KH} \mathrm{KH}_{2} \mathrm{PO}_{2} \mathrm{KPO}_{4}, \mathrm{pH}$ 6.2). Ax2 amoebae were grown in HL5 (Watts and Ashworth 1970) supplemented with $10 \mu \mathrm{g} / \mathrm{ml}$ penicillin $\mathrm{G}$ and $7 \mu \mathrm{g} / \mathrm{ml}$ streptomycin sulphate.

\subsection{Strains: Growth and development}

Ax2, an axenic strain of $D$. discoideum, is a commonly used surrogate for the wild type; the trishanku (triA $A^{-}$) mutant as well as Ax2 and trit $A^{-}$transformants expressing GFP have been described (Jaiswal et al. 2006). The cell counting factor mutants countin ${ }^{-}, c f 50^{-}$and $c f 45^{-}$were obtained from the Dictyostelium Stock Center, Northwestern University, USA. Cells of rit $^{-}$and the cell-counting factor (CF) mutants countin ${ }^{-}$, cf $50^{-}$and $c f 45^{-}$were grown in HL5 supplemented with $5-10 \mu \mathrm{g} / \mathrm{ml}$ blasticidin. Transformants (Ax2 Actin15-GFP and triA ${ }^{-}$Actin15GFP) were maintained in HL5 supplemented with $20 \mu \mathrm{g} / \mathrm{ml} \mathrm{G} 418$. Starvation was induced by spinning down cells at $300 \mathrm{~g}$ for $3 \mathrm{~min}$, suspending them in icecold KK2 and repeating the procedure twice. Freshly starved amoebae were plated at a density of $5 \times 10^{5}$ cells $/ \mathrm{cm}^{2}$ or $1 \times 10^{6} \mathrm{cells} / \mathrm{cm}^{2}$ on KK2 agar plates $(1.5 \%$ or $2 \%$ agar in KK2) to allow for development to proceed. The time of plating was taken as $0 \mathrm{~h}$.

\subsection{Mixing}

Cells were grown as shaken cultures in HL5 supplemented, if required, with $10 \mu \mathrm{g} / \mathrm{ml}$ G418. They were separated from nutrients when at a density of about $2 \times 10^{6}$ cells $/ \mathrm{ml}$ by washing them twice by centrifuging at $300 \mathrm{~g}$ for $3 \mathrm{~min}$ in ice-cold KK2. Cells were re-suspended at a density of $1 \times 10^{6} / \mathrm{ml}$ and incubated for $3 \mathrm{~h}$ in a $22^{\circ} \mathrm{C}$ shaker at $150 \mathrm{rpm}$; this was to take into account cell divisions in amoebae that happen to be in the late G2 phase of the mitotic cycle when starved (something that can lead to an increase of up to $20 \%$ in cell number). They were recounted, mixed in the desired ratio (using unlabelled Ax 2 cells and fluorescent trit $A^{-}$cells, or vice versa) and 
spread on KK2 agar plates at the required density. Plates were incubated in the dark at $22^{\circ} \mathrm{C}$; streaming was monitored after $8-9 \mathrm{~h}$, and the number of aggregates or fruiting bodies was counted after 1 or more days.

\subsection{Conditioned medium}

Conditioned medium (CM) was made from starved cells as described by Gomer et al. (1991). Ax2 and trit $A^{-}$cells were starved, re-suspended in KK2 buffer at a density of $1 \times 10^{7} \mathrm{cells} / \mathrm{ml}$ and kept shaking at $120 \mathrm{rpm}$ at $22^{\circ} \mathrm{C}$ for $20 \mathrm{~h}$. Cells were separated by centrifuging at $4^{\circ} \mathrm{C}$ and $800 \mathrm{~g}$ for $90 \mathrm{~s}$, and the supernatant was clarified by centrifugation at $8000 \mathrm{~g}$ for $15 \mathrm{~min}$ and $4^{\circ} \mathrm{C}$. The clarified supernatant or $\mathrm{CM}$ was used immediately. To see the effect of $\mathrm{CM}$ on aggregate size, Ax2 and trit ${ }^{-}$cells were starved on Whatman \#3 filters, which were placed on Whatman \#1 filters soaked in Ax2 CM or triA $A^{-} \mathrm{CM}$. Cells starved on Whatman \#1 filters soaked in KK2 buffer were used as a control. Alternatively, $1 \times 10^{6}$ cells $/ \mathrm{cm}^{2}$ cells were developed in submerged conditions in a $60 \mathrm{~mm}$ Petri dish containing $2.5 \mathrm{ml}$ of either $\mathrm{Ax} 2 \mathrm{CM}$ or trit ${ }^{-} \mathrm{CM}$ (as described previously by Okuwa et al. 2001). Cells submerged in $2.5 \mathrm{ml}$ of KK2 buffer were used as a control. The plates were incubated at $22^{\circ} \mathrm{C}$. Streaming was monitored after $8-9 \mathrm{~h}$ and aggregates were counted after 12-13 h.

\subsection{Spore counts}

Once fruiting bodies had formed, plates were kept at $22^{\circ} \mathrm{C}$ for 2-3 days. Fruiting bodies were scored according to their morphology. Following that, individual fruiting bodies were picked up with a needle, placed in a drop of water on a glass slide and a cover slip placed on top. The cover slip was tapped gently to disperse spores. These were then photographed under bright field and fluorescence at $60 \times$ using a Leica DM IRB inverted microscope. Fluorescent and nonfluorescent spores were counted from photographs. About 200-500 spores per fruiting body and 10-15 fruiting bodies were monitored in each experiment. The 1:1 mixing experiment was repeated five times and the other two mixes (1:4 and 4:1) thrice.

\subsection{Calculations and statistical analyses}

The rough approximation $1 / N=\pi r^{2}$ was used to convert $N$, the mean number of aggregates or fruiting bodies per unit area, to $r$, the mean radius of an aggregation territory. This formula assumes identical and uniformly distributed circular territories with each circle touching six others. It overestimates $r$ when aggregation streams break up as in tri $^{-}$.
Student's $t$-test for non-paired samples and one-way ANOVA were carried out using the 'Statistica' 99 Edition software or standard formulas available in $h t t p: / / w w w$. graphpad.com/quickcalcs/ttest1.cfm? Format $=S D$. $p$-values of $<0.05$ were considered statistically significant.

\section{Results}

\section{1 triA $^{-}$aggregates are smaller than normal}

tri $A^{-}$aggregation streams form as in the parental $\mathrm{Ax} 2$ but break up after some time. The reasons could be many; earlier work points to defective intercellular adhesion as a possible cause (Jaiswal et al. 2006). Consequently, triA $^{-}$aggregation territories are significantly smaller than those of Ax2 (figure 1A). Ax2 cells formed $\sim 85$ aggregates $/ \mathrm{cm}^{2}$ and triA $^{-}$ formed significantly more, i.e. $\sim 132$ aggregates $/ \mathrm{cm}^{2}$, at two different plating densities $\left(5.0 \times 10^{5}\right.$ cells $/ \mathrm{cm}^{2}$ and $1.0 \times 10^{6}$ cells $/ \mathrm{cm}^{2} ; t$-test for non-paired samples; $p<0.05, n=3$; figure $1 \mathrm{~A}, \mathrm{~B})$.

Thus, the average triA $A^{-}$aggregate covers a smaller territory than an Ax2 aggregate. The mean radius of a territory works out to $\sim 0.63 \mathrm{~mm}$ for Ax2 and $\sim 0.49 \mathrm{~mm}$ for tri $A^{-}$. Both figures are based on plate-wide counts and the trit $A^{-}$value is an overestimate of the true radius (because we have not taken into account the fact that stream break-up leads to a clumped distribution of aggregates). The Ax2 figure is similar to what Bonner and Dodd (1962) found in wild-type $D$. mucoroides and slightly less than half of what they estimated for $D$. discoideum (they worked with wildtype cells, not Ax2).

\subsection{Aggregation streams break up in Ax2+ triA chimaeras too}

Jaiswal et al. (2006) found that when mixed in 1:1 ratio, Ax2 and tri $^{-}$cells co-aggregated freely, but the chimaeric aggregation streams fragmented in a manner similar to triA streams. Evidently the triA ${ }^{-}$component prevented streams from going on to complete the process of aggregation. We followed the development after mixing freshly starved GFPtagged tri $A^{-}$cells with Ax2 cells in a 1:9 ratio. Even though triA $^{-}$cells were in a minority, chimaeric aggregation streams broke up during mid to late aggregation (figure 2). The appearance of discrete aggregation mounds along diverging lines showed that the cells in them had been a part of streams that were radiating outwards from a common centre (figure 2C). Control mixes of GFP-tagged Ax2 cells and unlabelled Ax2 cells aggregated normally without any signs of streams breaking up (figure 2A). The fact that a small minority of tri $^{-}$cells can cause largely Ax2 aggregation 
(A)

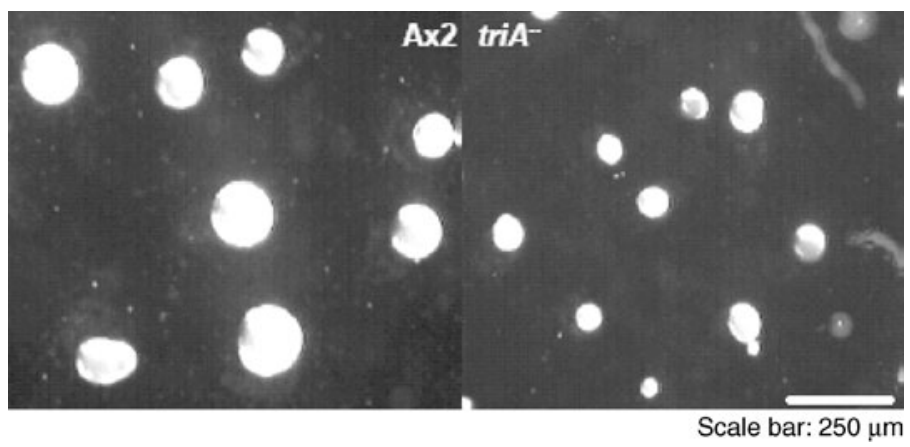

(B)

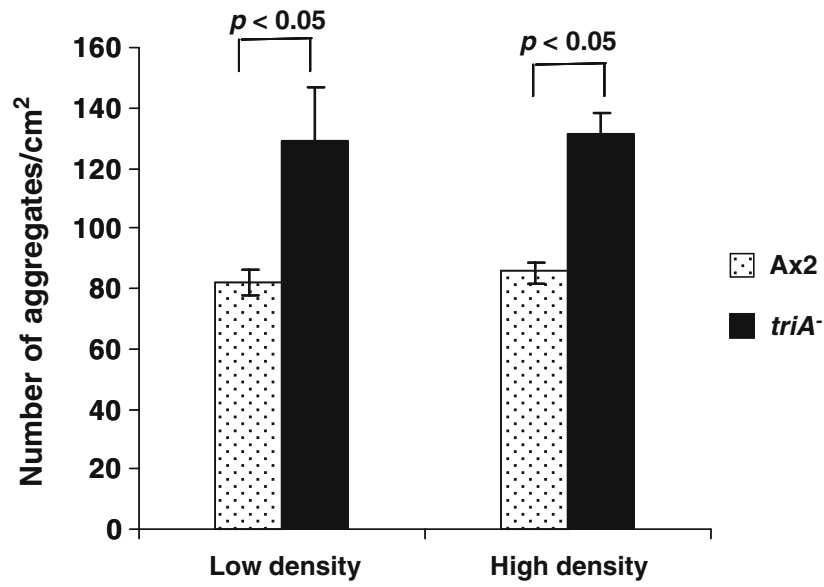

Figure 1. Aggregation territory sizes in Ax2 and triA $A^{-}$. (A) trit $A^{-}$aggregates are smaller than those of Ax2 when plated at the same density $\left(5 \times 10^{5}\right.$ cells $\left./ \mathrm{cm}^{2}\right)$. (B) Aggregate density is inversely related to aggregate size. tri $A^{-}$forms significantly more aggregates than Ax2 when plated at two different densities $\left(5 \times 10^{5}\right.$ cells $/ \mathrm{cm}^{2}$ and $1 \times 10^{6}$ cells $/ \mathrm{cm}^{2} ; t$-test for non-paired samples, $\left.p<0.05, n=3\right)$.

streams to break up hints that under these conditions rather than direct cell-cell contact, an extracellular factor or factors could be responsible for maintaining the integrity of streams.

\subsection{Conditioned medium from triA $A^{-}$lowers Ax2 aggregate size by causing streams to break up}

We tested for the role of extracellular factors in stream break up by monitoring the development of $\mathrm{Ax} 2$ and trit $A^{-}$cells in the presence of $\mathrm{CM}$ obtained from cells of either strain. In the presence of $\mathrm{Ax} 2-\mathrm{CM}, \mathrm{Ax} 2$ cells formed aggregates whose territory size was about the same as those formed in the presence of buffer (figure 3): the number of aggregates formed per $\mathrm{cm}^{2}$ by $\mathrm{Ax} 2$ cells developing in buffer was 102.2 \pm 3.5 ; and when developing in Ax2-CM, this number was $117.2 \pm 4.8$. When $\mathrm{Ax} 2$ cells developed in the presence of $t r i A^{-}-\mathrm{CM}$, the aggregates were noticeably smaller in size and so there were many more of them per unit area, namely, $188.4 \pm 3.5 / \mathrm{cm}^{2}$ (figure 3). This is significantly higher than the value for Ax2 cells developing in buffer or in $\mathrm{Ax} 2-\mathrm{CM}$ (mean $\pm \mathrm{SD}, n=4$; one-way ANOVA, $p<0.005)$.
Irrespective of whether they developed in buffer, Ax2$\mathrm{CM}$ or $\operatorname{tri}^{-}{ }^{-} \mathrm{CM}$, tri $A^{-}$cells formed aggregates that were smaller than those of Ax2 (figure 3). The number of triA aggregates per $\mathrm{cm}^{2}$ was $138.9 \pm 5.9$ when cells were starved in buffer; they were $198.9 \pm 7.7$ and $183.4 \pm 18.2$, respectively, when triA $^{-}$cells were starved in the presence of $\mathrm{Ax} 2-\mathrm{CM}$ or $\operatorname{triA}^{-}-\mathrm{CM}$ (figure 3 ). In the presence of Ax2$\mathrm{CM}$, tri $A^{-}$formed significantly more aggregates than $\mathrm{Ax} 2$, but the number was comparable to that for $\mathrm{Ax} 2$ cells developing in $\operatorname{triA}^{-}-\mathrm{CM}$ (mean $\pm \mathrm{SD}, n=4$; one-way ANOVA ; $p<0.005, n=4)$.

\subsection{Conditioned medium works independently of counting factor}

A complex of released polypeptides collectively known as CF and the products of the countin 2 and countin 3 genes regulate group size in $D$. discoideum. CF is overproduced in the smlA mutant and results in smaller-than-normal aggregates (Brock et al. 1996; Roisin-Bouffay et al. 2000). Loss of function mutations in countin 2 and countin 3 also lead to a decrease in aggregate size (Okuwa et al. 2001, 2002; Katayama et al. 


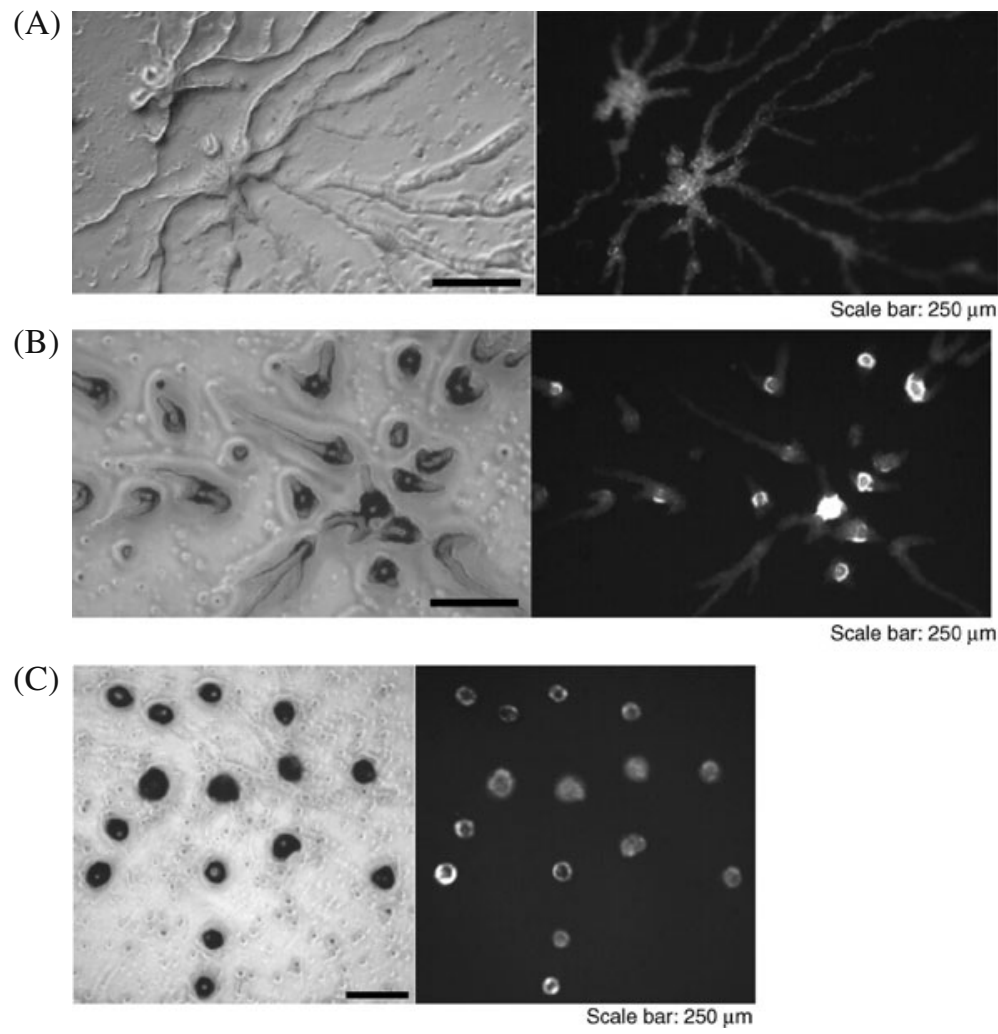

Figure 2. Aggregation streams break up in mixed groups of Ax 2 and triA ${ }^{-}$. (A) Ax2 cells (90\%) mixed with a minority of GFP-tagged Ax2 cells (10\%) aggregate normally via long, continuous streams. (B) When Ax2 cells (90\%) are mixed with a minority of GFP-tagged tri $A^{-}$cells (10\%), streams break up during mid to late aggregation. (C) The mounds formed after stream break up in Ax2 (90\%): triA $(10 \%)$ chimaeras are seen in rows depicting the remanants of broken streams (bright field images on left, fluorescence on right).

2003). On the contrary, because a gene encoding a component of the multiprotein-counting factor complex is knocked out in the countin ${ }^{-}, c f 50^{-}$and $c f 45^{-}$strains, they show an increase in aggregate size (Brock et al. 2002, 2003). Unlike the product of the countin 2 or countin 3 genes, the extracellular factor produced by $s m l A$ null cells is well characterized. For this reason we tested whether the stream break up in tri $A^{-}$cells could also be due to an overproduction of $\mathrm{CF}$.

Countin $^{-}, c f 50^{-}$and $c f 45^{-}$cells were starved in the presence of Ax2-CM or $\operatorname{tri}^{-}-\mathrm{CM}$. If, like $s m l A$, tri $A^{-}$ is indeed an overproducer of $\mathrm{CF}$, the counting factor mutants should form smaller aggregates in the presence of triA ${ }^{-}$-CM compared with Ax2-CM (Brock and Gomer 1999). Cells of all three mutants did form many more, and so smaller, aggregates in Ax2-CM or tri $A^{-}-\mathrm{CM}$ than in buffer. However, the numbers in the two CMs were comparable (figure 4; one-way ANOVA, $p>0.05, n=3$ ). We infer that over and above defective cell-cell adhesion (Jaiswal et al. 2006; Mujumdar et al. 2009), diffusible factors that act independently of CF cause $t r i A^{-}$streams to fragment

\subsection{Fruiting body morphologies in Ax2+triA ${ }^{-}$chimaeras}

When freshly starved amoebae of Ax2 and tri $^{-}$are mixed in a 1:1 ratio, the two strains aggregate, develop in concert and form compound fruiting bodies of a 'double-decker' (DD)-type (Jaiswal et al. 2006). We repeated those experiments and carried out others with 4:1 and 1:4 initial mixing ratios of Ax2 to triA $A^{-}$amoebae. Three types of fruiting body were seen (figure 5). In some the spore mass was right at the top of the stalk, as in the wild type (and in Ax2); in others it appeared to have stopped midway up the stalk, as in trishanku (tri $A^{-}$-type). The remaining fruiting bodies consisted of a stalk with two spore masses, one at the apical terminus, as in Ax2 and the other midway, as in tri $A^{-}$. As before, we term the latter DD-types.

The relative proportions of the three types of fruiting bodies $\left(\mathrm{Ax} 2:\right.$ tri $\left.A^{-}: \mathrm{DD}\right)$ varied with the mixing ratio $\left(\mathrm{Ax} 2: t r i A^{-}\right.$; table 1 and figure 6). Most fruiting bodies were of the Ax2 or tri $A^{-}$-type when the initial mix of genotypes was 1:1 (means: 45.7\% Ax2-type, 47.2\% tri $A^{-}$-type and 7.1\% DD-type). When the ratio was $4: 1$, the mean proportions were $87.88 \%$ 

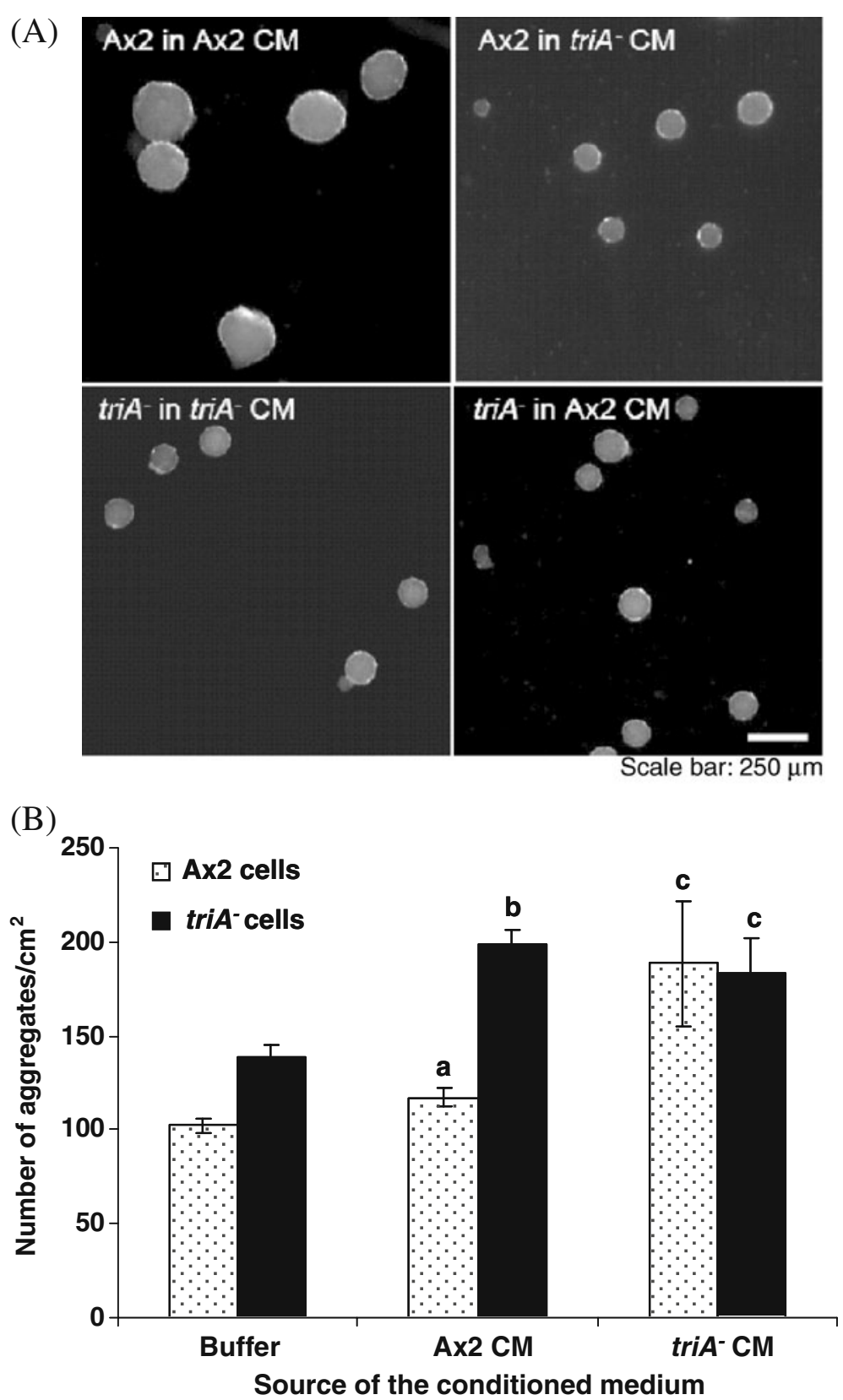

Figure 3. Ax2 and tri $A^{-}$aggregate sizes in Ax2 or tri $A^{-}$conditioned medium (CM). (A) Ax2 forms smaller aggregates when starved in

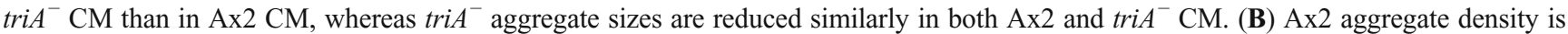
higher in tri $^{-} \mathrm{CM}$ than in Ax2 CM (a vs c; $p<005$ ). The number of aggregates formed by Ax2 and triA ${ }^{-}$cells in Ax2 $\mathrm{CM}$ is also significantly different (a vs $\mathrm{b} ; p<0.005$ ). The difference in aggregate density between $\mathrm{Ax} 2$ and tri $^{-}$cells developing in $t r i A^{-} \mathrm{CM}$ is insignificant $(p>0.05)$. All $p$-values are based on one-way ANOVA, $n=4$.

(Ax2-type), 4.81\% (tri-type) and 7.29\% (DD-type). When the mixing ratio was $1: 4$, DD-types were absent; essentially all fruiting bodies were of the tri-type. We estimated the relative proportions of Ax2 and $t_{r i A^{-}}$spores in chimaeric fruiting bodies in order to see whether there was a spatial separation between the genotypes. Earlier we had said that the upper mass in DD-type fruiting bodies was made of Ax2 cells and the lower spore mass of triA cells; we went on to infer that the location of the spore mass depended on cell-autonomous properties (Jaiswal 


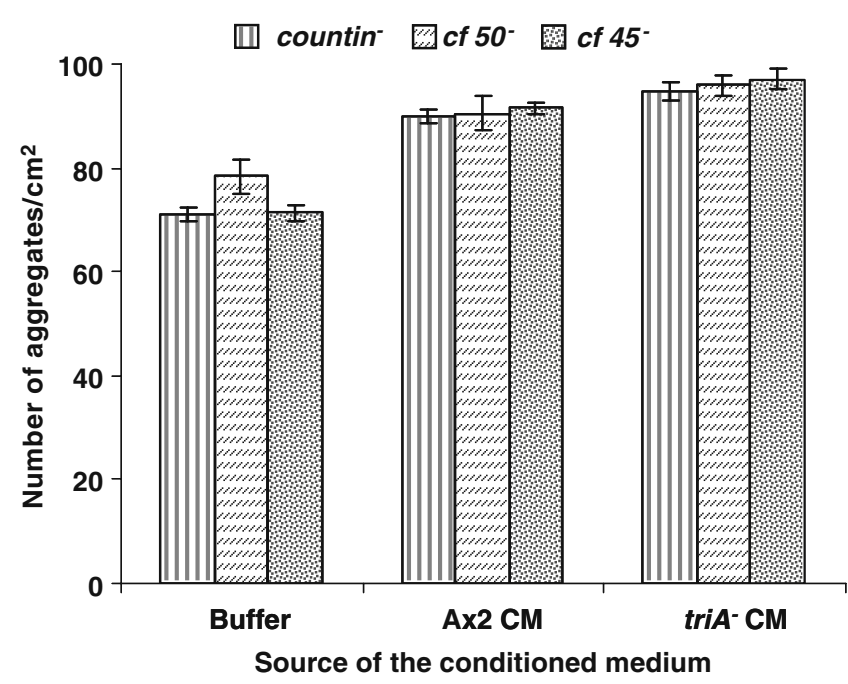

Figure 4. Countin ${ }^{-}$, cf $50^{-}$and $c f 45^{-}$aggregate sizes in buffer, $\mathrm{Ax} 2 \mathrm{CM}$ or triA $A^{-} \mathrm{CM}$. Countin ${ }^{-}$, cf $50^{-}$and $c f 45^{-}$cells form more aggregates $/ \mathrm{cm}^{2}$ in $\mathrm{Ax} 2$ or $\mathrm{triA}^{-} \mathrm{CM}$ than in buffer. However, aggregate densities between the two $\mathrm{CMs}$ are not significantly different ( $p>0.05$; one-way ANOVA, $n=3$ ).

et al. 2006). As will be seen, that needs to be corrected: we had relied on the gross pattern of fluorescence seen after mixing fluorescent and non-fluorescent cells and had not monitored individual spores.

\subsection{Spatial distribution of spore genotypes in chimaeric fruiting bodies}

The distribution of Ax2 and triA $A^{-}$spores across the three types of fruiting bodies at different Ax2:triA $A^{-}$mixing ratios displayed unexpected features (table 2 and figure 7).

When the mixing ratio was $4: 1$, slightly more than the expected $(80 \%)$ spores belonged to $\mathrm{Ax} 2$ (the means ranged from $81.03 \%$ to $87.85 \%$ depending on the fruiting body type). Also, the difference in frequency of Ax2 spores between Ax2-type and tri-type fruiting bodies was just

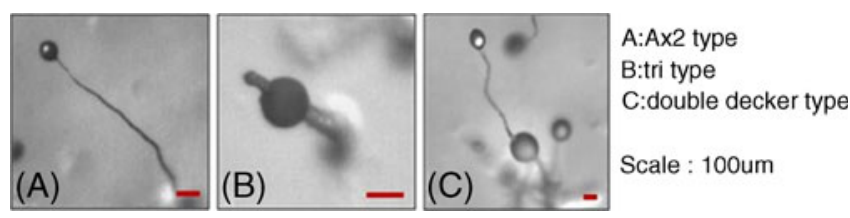

Figure 5. Fruiting body morphologies in Ax2+trishanku mixtures. (A) Ax2-type; (B) tri-type; (C) Double-decker-type. Scale bar: $100 \mu \mathrm{m}$. significant $(p=0.05)$; that between the upper and lower spore masses in DDs was not $(p=0.11)$.

With a 1:4 mixing ratio, Ax2 spores were somewhat fewer than expected $(\sim 15 \%$ against the expected $20 \%$, the difference being not significant, $p=0.3$ ), and almost only tritype fruiting bodies were seen. Thus, irrespective of the fruiting body morphology, at both 4:1 and 1:4 mixing ratios, the output (proportion of $\mathrm{Ax} 2$ or $\mathrm{tri}^{-}$spores) corresponded approximately to the input (proportion of Ax2 or triA amoebae) but with a bias in favour of the majority component.

In fruiting bodies formed from 1:1 mixes, Ax2 spores made up a mean of $70.71 \%$ of all spores in Ax2-type fruiting bodies and $34.71 \%$ in trit $A^{-}$-type fruiting bodies; the difference is statistically significant ( $t$-test, $p=0.0002$ ). In DD-type fruiting bodies, both genotypes were present in both spore masses but $\mathrm{Ax} 2$ spores were in a majority (mean, $69.84 \%$ ) in the upper spore mass and a minority (mean, $34.43 \%$ ) in the lower spore mass. The fraction of spores in the upper spore mass of DD-type fruiting bodies that belong to Ax2 is comparable to that in Ax2-type fruiting bodies $(p=0.43)$; correspondingly, the fraction of triA $A^{-}$spores in the lower spore mass is comparable to that in tri-type fruiting bodies $(p=0.66)$. There is a significant difference between the upper and lower spore masses of DD-type fruiting bodies in the fraction of spores that belong to $\operatorname{Ax} 2(t=7.4838, d f=14$, $p<0.0001)$. Interestingly, when counts from the upper and lower masses in DD-type fruiting bodies from 1:1 mixes are added, the ratio of genotypes reverts to $1: 1$.

\subsection{Mixing controls}

Two sorts of mixing controls were performed. Mixes between cells of the same genotype were carried out in order to verify that the GFP reporter did not affect the results. Next, plates were incubated with 1:1 mixes of starved Ax2+Ax2-GFP, triA ${ }^{-}+$triA $^{-}$-GFP, Ax2+ triA ${ }^{-}$-GFP or triA $^{-}+\mathrm{Ax} 2$-GFP amoebae. After aggregation was over and slugs had formed, individual slugs were transferred to a glass slide containing a drop of $\mathrm{KK} 2$, mechanically disrupted, thoroughly stirred with a thin metal wire, and fluorescent and non-fluorescent cells counted. About 400 cells were counted in one slug and counts from approximately nine slugs pooled in each batch. From eight such batches the absolute difference in proportions between labelled and unlabelled cells was $3.80 \pm 4.47 \%$ (mean $\pm \mathrm{SD}$ ), in effect zero. If we restrict ourselves to the $\mathrm{Ax} 2+$ triA $^{-}$ chimaeras, the minority strain in slugs was $t r i A^{-}$in one case and $\mathrm{Ax} 2$ in the remaining three. Its mean representation was $47.05 \%$, i.e. not significantly different from $50 \%(p=0.14$, two-tailed $t$-test). We took this to mean that even in the smaller-than-normal aggregates resulting from stream break- 
Table 1. Fruiting body morphologies (mean \pm s.d.) at various mixing ratios of Ax 2 and trishanku amoebae

\begin{tabular}{|c|c|c|c|}
\hline \multirow[b]{3}{*}{ Fruiting body morphology } & \multicolumn{3}{|c|}{ Initial mixing ratio $\left(\mathrm{Ax} 2:\right.$ tri $\left.^{-}\right)$} \\
\hline & $4: 1$ & $1: 1$ & $1: 4$ \\
\hline & \multicolumn{3}{|c|}{ No. of fruiting bodies $/ \mathrm{cm}^{2}$} \\
\hline Ax2-type & $87.88 \pm 3.50$ & $45.64 \pm 12.33^{*}$ & $0.30 \pm 0.41$ \\
\hline Tri-type & $4.81 \pm 2.89$ & $47.33 \pm 13.53 *$ & $99.69 \pm 0.30$ \\
\hline Double-decker-type & $7.29 \pm 0.65$ & $7.02 \pm 4.28$ & 0 \\
\hline Fruiting bodies counted & 3789 & 12380 & 4287 \\
\hline No. of experiments & 5 & 20 & 6 \\
\hline
\end{tabular}

The total number of fruiting bodies counted in each category and the number of independent counts are given. In the last column (1:4) 13 fruiting bodies were of the Ax2 type and 4274 were of the tri-type. All differences except the one between the figures marked by an asterisk (*) are significant at the $99 \%$ level or better.

up, cells remained mixed in their initial proportions in slugs and, by extension, in the fruiting bodies that resulted from them.

As we have seen, experimental outcomes varied qualitatively between chimaeras of the same composition. Even if overall proportions were as expected, there could have been variations in the spatial segregation of presumptive spore and stalk cells within otherwise similar aggregates and slugs. Along with a 'majority wins' rule, that could have led to the differences in relative spore counts between Ax2-type and tri-type fruiting bodies. A number of facts make this unlikely. The striking difference in the pattern of findings between chimaeras of the extreme mixing ratios $(4: 1$ and $1: 4$; table 1) is one. Also, DD-type fruiting bodies result from both 4:1 and 1:1 mixes, but the Ax2:triA ${ }^{-}$ratio in their spores is very different (table 2). The most parsimonious explanation of all these observations is that (a) initial mixing ratios are maintained in slugs and fruiting bodies and (b) sorting out of cell types within mixes is not a sufficient explanation for the results.

\section{Discussion}

Since the aim was to distinguish between cellautonomous and cell-non-autonomous traits in D. discoideum groups, we should have monitored individual cell behaviour. We were unable to do so because the traits of interest (morphology of aggregation streams and fruiting bodies) were expressed only in cell groups. Two facts helped in overcoming the difficulty. First, traits were invariant within genetically homogeneous $\mathrm{Ax} 2$ or triA $^{-}$ groups and different between groups; there was no range variation (Bonner 1965). So, we could think of the traits as characteristic of genotypes, i.e. group behaviour was associable with genotype-specific cell behaviour. Second, and in contrast, chimaeras exhibited range variation: the expression of a trait varied significantly between similar chimaeric groups. This helped us to exploit chimaeras for drawing inferences regarding interactions between different phenotypes, i.e. inferences that applied also to

\section{Fruiting body types at different mixing ratios}
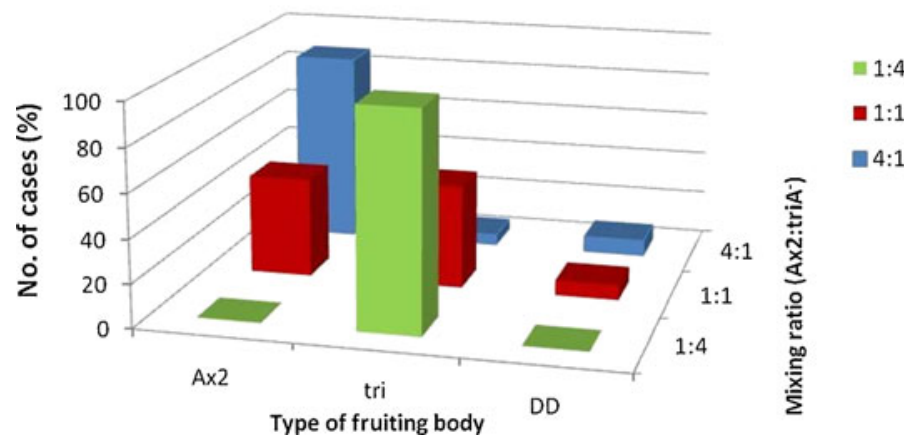

Figure 6. Distribution of fruiting body morphologies in Ax2:triA $A^{-}$mixes. Only mean values are shown for the sake of clarity. Except for the Ax2-type and tri-type fruiting bodies in the 1:1 mix, all differences are significant at the 99\% level. See table 1 and text for details. 
Table 2. Percentage (\%) of Ax 2 spores across the three types of fruiting bodies after freshly starved Ax2 and trishanku amoebae were combined in various mixing ratios

\begin{tabular}{lccc}
\hline & & \multicolumn{2}{c}{ Initial mixing ratio (Ax2:tri $\left.A^{-}\right)$} \\
\cline { 2 - 4 } & $4: 1$ & $1: 1$ \\
\cline { 2 - 4 } Fruiting body morphology & & Fraction of Ax2 spores (\%) \\
\hline Ax2-type & $87.85 \pm 3.63$ & $70.71 \pm 11.98$ & 0 \\
Tri-type & $83.03 \pm 2.79$ & $34.17 \pm 9.55$ & $14.88 \pm 4.24$ \\
Double-decker (upper sorus) & $81.03 \pm 4.51$ & $69.84 \pm 7.01$ & 0 \\
Double-decker (lower sorus) & $85.51 \pm 3.04$ & $34.43 \pm 6.67$ & 0 \\
\hline
\end{tabular}

In each case the proportion of trishanku spores is 100 minus the number shown. Differences between proportions of Ax 2 and triA ${ }^{-}$spores are significant in all cases. In addition, in the 1:1 mix each genotype is represented in the upper and lower sori at a frequency that is significantly different from $50 \%(p<0.01$, unpaired $t$-test $)$.

genetically homogeneous groups. Figure 8 indicates a schematic framework within which we view the findings.

\subsection{Autonomy and non-autonomy}

If cells behaved in accordance with their respective genotypes alone, the number of aggregates per unit area in Ax2-tri $A^{-}$mixtures would be a weighted mean of the Ax2 and tri $^{-}$values. This was clearly not so (see figures 1 and $2 \mathrm{~B}$, where triA ${ }^{-}$cells are $10 \%$ of the total). The CM experiments showed that even though both Ax2-CM and tri $^{-}$-CM caused an increase in the number of aggregates, tri $^{-}$cells were the more potent producers of the relevant factor(s) as well as the more sensitive responders (figure $3 \mathrm{~A}, \mathrm{~B}$ ). We infer that territory size in chimaeric aggregate depends on autonomous properties based on intrinsic strain sensitivities and non-autonomous effects due to extracellular diffusible factors.

Autonomy in fruiting bodies could mean that Ax2 spores ascended to the top of the stalk in chimaeras and tri $A^{-}$spores remained midway. Or, a fruiting body of one species could form above that of the other (as in interspecies grafts; Bonner and Adams 1958). Instead, most chimaeric fruiting bodies were of the Ax2- or tri-type (figure 5 and table 1), with cells of both genotypes in each spore mass. DD fruiting bodies drive home the point nicely. Upper and lower spore masses in DD-type fruiting bodies formed by 1:1 mixes have substantial representations of each genotype, with more Ax2 cells than expected in the upper spore mass and more tri $A^{-}$cells than expected in the lower spore mass $(p<0.001$ in each case; table 2). The morphology and composition of chimaeric fruiting bodies too shows autonomous and non-autonomous factors at work. The basis of nonautonomy is communication via the production of diffusible factors $(\mathrm{CM})$ and, positively correlated with their production, sensitivity to the factors. These two new pleiotropic effects of the tri gene are comparable to those of the $l s r$ gene, which shows similarly mediated effects with regard to its influence on stalk induction (Parkinson et al. 2011). We do not know

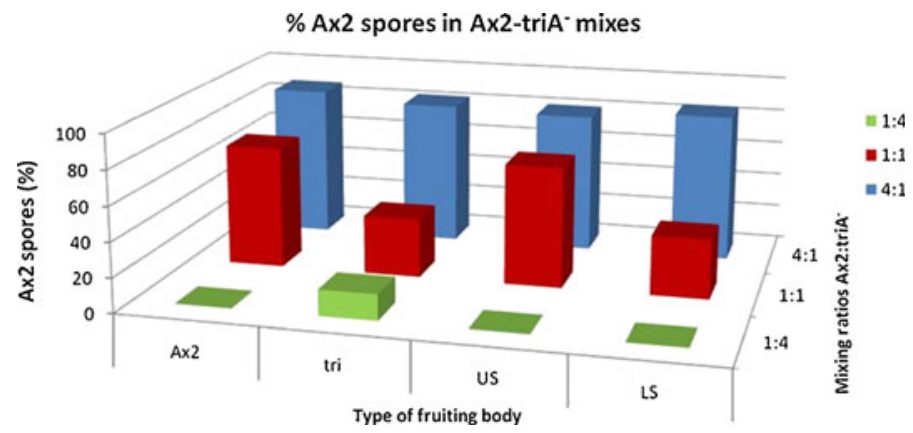

Figure 7. Distribution of genotypes across fruiting body types in Ax2:triA $A^{-}$mixes. Only mean values are shown for the sake of clarity. Except for the cases in which no Ax2 spores were seen, all differences are significant at the $99 \%$ level or better (US and LS, upper spore mass and lower spore mass in double-decker-type fruiting bodies). See table 2 and text for details. 


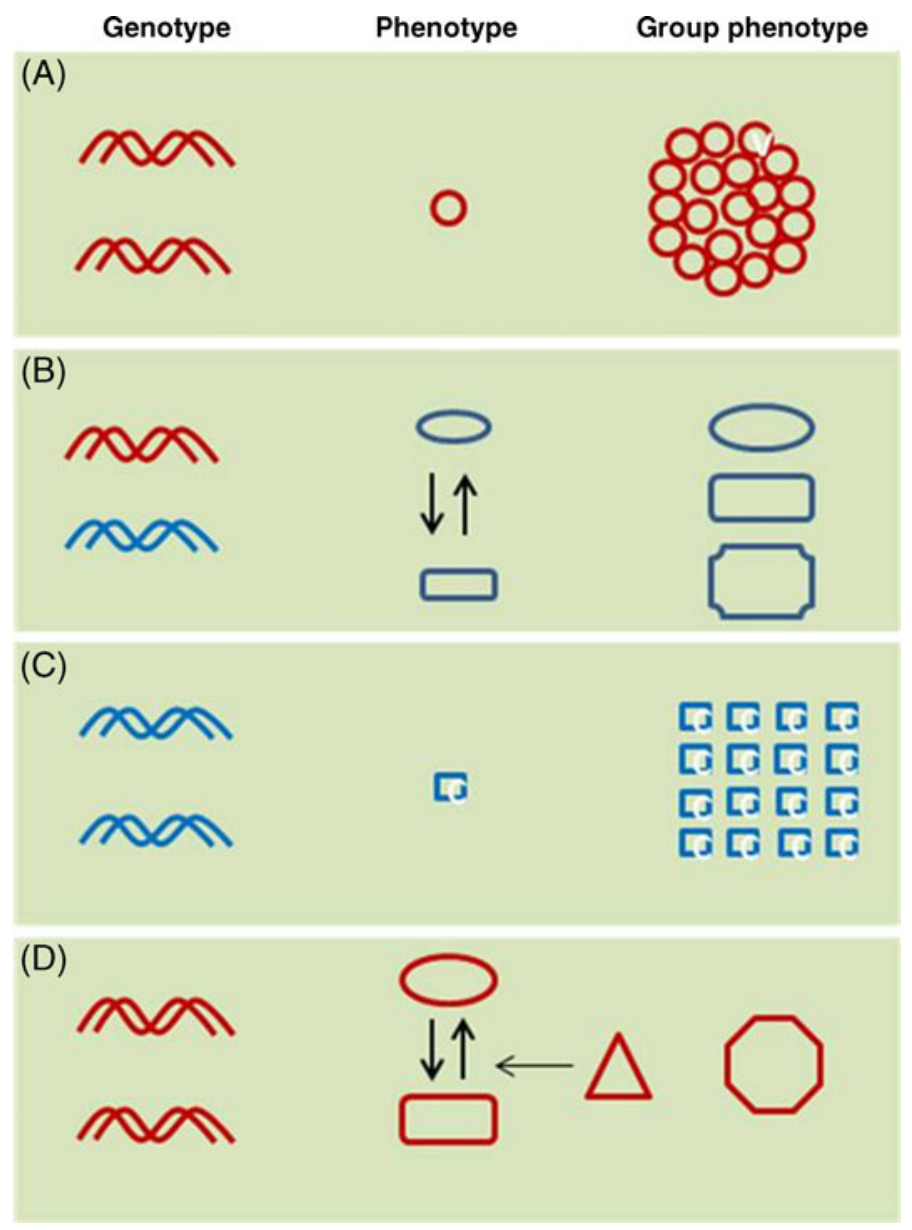

Figure 8. Comparison between social groups and multicellular development. (A, C) Groups formed by genetically identical units whose phenotypes do not interact; the group phenotype is a 'sum' of individual phenotypes; (B) group formed by heterogeneous units of the same genotypes as in (A) and (C), but the phenotypes influence each other (arrows) and so are different from the ones in situations (A) and (C); development (group behaviour) may or may not be canalised; if not, the group phenotype can resemble that of either homogeneous group or something new; (D) group formed by genetically homogeneous units with phenotypic plasticity; phenotypes interact (vertical arrows) in a manner that is influenced by other phenotypes (the bystander effect: horizontal arrow); development (group behaviour) is canalised; the group phenotype is not easily predictable from that of the units. The light green background stands for the environment and is meant as a reminder that genotype $\rightarrow$ phenotype links are strongly environment-dependent.

whether the same set of factors in CM mediate stream breakup, fruiting body morphology and differentiation.

\subsection{Interacting phenotypes}

The heights of the green, red and blue bars in the three fruiting body types (figures 6 and 7) show that not only do the relative proportions of spore genotypes vary with the mixing ratio but that the pattern of variation depends on the type of fruiting body. The implication is that the outcomes depend on how phenotypes interact. Thus, fruiting body morphology and spore composition are traits that exhibit incomplete penetrance to an extent that depends on the genetic background. Buttery et al. (2010) came to a similar conclusion after mixing pairs of natural (wild-type) isolates of $D$. discoideum: direct genetic effects, indirect genetic effects and epistatic interactions between genotypes all influenced the outcome.

triA $A^{-}$is the dominant partner in $\mathrm{Ax2}$-triA $A^{-}$interactions. For one thing, the number of aggregates per unit area in mixes obviously leans towards the tri $A^{-}$value (figures 1 and $2 \mathrm{~B}$, where tri $A^{-}$cells make up $10 \%$ of the total). So also, $20 \%$ of triA $^{-}$cells were sufficient to make many fruiting bodies adopt either the tri-type or DD-type phenotype, whereas in the reciprocal mix, when $20 \%$ of the chimaera consisted of Ax2 cells, hardly any displayed the Ax2-type phenotype (table 1). 
Cell-cell interactions in CSM groups involve a further intricacy, namely, a nonlinear effect that requires a group of at least three genotypes to be perceived (Kaushik et al. 2006): the relative sporulation efficiency of amoebae belonging to two genotypes is affected by the presence of a third genotype (Kaushik et al. 2006; Khare et al. 2009). This is a 'bystander effect': the behaviour of one individual towards another is modulated by the presence of a bystander. The bystander effect has been discovered in cancer tissues (Mesnil et al. 1996) and must occur in normal development too.

\subsection{Breakdown of canalisation}

As we have argued, incomplete mixing between strains or differential segregation within slugs is unlikely to be a satisfactory explanation for why more than one type of fruiting body results from comparable mixes (table 1). We rather feel that the different fruiting body morphologies point to a lowered precision of development in chimaeras that lack a history of co-evolution. Canalisation (Waddington 1942) often breaks down in mutants because they have not been selected for developmental reliability. Similarly, destabilisation of morphogenetic pathways in Ax2-tri $A^{-}$chimaeras could be behind the increased range of developmental outcomes. On the other hand, coexisting wild-type genotypes of a CSM species would have a shared evolutionary past as members of multiclonal groups (Kawli and Kaushik 2001) and would be expected to show canalised development. The stability of a heterogeneous social group, too, may depend on a history of co-evolution.

\subsection{Multicellular development and social groups}

Many influences intervene during multicellular development between gene expression and phenotype. Besides environmental factors, they include the other allele at the same locus (in diploids) and alleles at other loci. In social groups, too, the phenotype of an individual is influenced by its own genotype and by the phenotypes, and so genotypes, of the individuals with which it interacts. The coherent functioning of a group of cells or multicellular individuals depends on both autonomous and nonautonomous properties exhibited by its members. Phenotypic plasticity and reciprocal interactions between phenotypes are all-important in both situations (figure 8; Gilbert and Epel 2008; Nanjundiah and Sathe 2011). This means that whether or not group members share genes by common descent, the frequently made assumption of genotype-specific traits (all the more so, of gene-specific traits) is liable to be wrong in both situations. Dominance, penetrance, epistasis and autonomy are commonly used terms in the genetic analysis of development. It should cause no surprise that they can aid our understanding of social behaviour too. As the quotation at the beginning suggests, cautious use of the same language for describing multicellular development and social behaviour may lead to interesting insights.

\section{Acknowledgements}

We are grateful to John Bonner, Stuart Newman and Chris Thompson for commenting on an earlier version of the text and to an anonymous reviewer for reminding us of the quotation that comes at the head of the article. Thanks are due to JK Roy for providing an opportunity to present these results at a symposium on the theme 'Functional Genomics: Challenges and Prospects' held at the Banaras Hindu University, India. This work was supported by funds from a UGC special assistance programme.

\section{References}

Babu P and Bhat SG 1986 Autonomy of the wingless mutation in Drosophila melanogaster. Mol. Gen. Genet. $205483-486$

Bonner JT 1958 The evolution of development (UK: Cambridge University Press)

Bonner JT 1965 Size and cycle (Princeton: Princeton University Press) Bonner JT 1967 The cellular slime molds 2nd edition (Princeton: Princeton University Press)

Bonner JT and Adams MS 1958 Cell mixtures of different species and strains of cellular slime molds. J. Embryol. Exp. Morphol. 6 $346-356$

Bonner JT and Dodd MR 1962 Aggregation territories in the cellular slime molds. Biol. Bull. 122 13-24

Brock DA and Gomer RH 1999 A cell-counting factor regulating structure size in Dictyostelium. Gene. Dev. 13 1960-1969

Brock DA, Buczynski G, Spann TP, Wood SA, Cardelli J and Gomer RH 1996 A Dictyostelium mutant with defective aggregate size determination. Development 122 2569-2578

Brock DA, Hatton RD, Giurgiutiu DV, Scott B, Ammann R and Gomer RH 2002 The different components of a multisubunit cell number-counting factor have both unique and overlapping functions. Development 129 3657-3668

Brock DA, Hatton RD, Giurgiutiu DV, Scott B, Jang W, Ammann R and Gomer RH 2003 CF45-1, a secreted protein which participates in Dictyostelium group size regulation. Euk. Cell 2 788-797

Buttery NJ, Thompson CRL and Wolf JB 2010 Complex genotype interactions influence social fitness during the developmental phase of the social amoeba Dictyostelium discoideum. J. Evol. Biol. 23 1664-1671

Fortunato A, Strassmann JE, Santorelli L and Queller DC 2003 Co-occurrence in nature of different clones of the social amoeba, Dictyostelium discoideum. Mol. Ecol. 12 1031-1038

Gilbert SF 2000 Developmental biology 6th edition (Sunderland, MA: Sinauer Associates Inc.) 
Gilbert S and Epel D 2008 Ecological developmental biology: Integrating epigenetics, medicine, and evolution (USA: Sinauer)

Gilbert OM, Queller DC and Strassmann JE 2009 Discovery of a large clonal patch of a social amoeba: implications for social evolution. Mol. Ecol. 18 1273-1281

Gomer RH, Yuen IS and Firtel RA 1991 A secreted 80x10(3) Mr protein mediates sensing of cell density and the onset of development in Dictyostelium. Development 112 269-278

Jaiswal JK, Mujumdar N, Macwilliams HK and Nanjundiah V 2006 Trishanku, a novel regulator of cell-type stability and morphogenesis in Dictyostelium discoideum. Differentiation $\mathbf{7 4}$ 596-607

Katayama T, Takana A, Okuwa T, Issaeva M, Masamune Y, Kodaira K and Yasukawa H 2003 Dictyostelium discoideum (countin3-) forms small fruiting bodies. Microbes Environ. 18 223-226

Kaushik S, Katoch B and Nanjundiah V 2006 Social behaviour in genetically heterogeneous groups of Dictyostelium giganteum. Behav. Ecol. Sociol. 59 521-530

Kawli T and Kaushik S 2001 Cell fate choice and social evolution in Dictyostelium discoideum. J. Biosci. 26 130-133

Kessin RH 1997 The evolution of the cellular slime molds: Dictyostelium - A model system for cell and developmental biology (Universal Academy Press)

Khare A, Santorelli LA, Strassmann JE, Queller DC, Kuspa A and Shaulsky G 2009 Cheater-resistance is not futile. Nature (London) 461 980-982

Le Douarin N and Teillet M 1974 Experimental analysis of the migration and differentiation of neuroblasts of the autonomic nervous system and of neuroectodermal mesenchymal derivatives using a biological cell marking technique. Dev. Biol. 41 162-184

Mesnil M, Piccoli C, Tiraby G, Willecke K and Yamasaki H 1996 Bystander killing of cancer cells by herpes simplex virus thymidine kinase gene is mediated by connexins. Proc. Natl. Acad. Sci. USA 93 1831-1835

Mintz B and Silvers WK 1967 'Intrinsic' immunological tolerance in allophenic mice. Science 158 1484-1486

Morata G and Lawrence PA 1977 The development of wingless a homeotic mutation of Drosophila. Dev. Biol. 56 227-240
Mujumdar N, Inouye K and Nanjundiah V 2009 The trishanku gene and terminal morphogenesis in Dictyostelium discoideum. Evol. Dev. 11 697-709

Nanjundiah V and Sathe S 2011 Social selection and the evolution of cooperative groups: The example of the cellular slime moulds. Integr. Biol. 3 329-342

Okuwa T, Katayama T, Takano A, Kodaira K and Yasukawa H 2001 Two cell-counting factors regulate the aggregate size of the cellular slime mold Dictyostelium discoideum. Devel. Growth Differ. 43 735-744

Okuwa T, Katayama T, Takano A and Yasukawa H 2002 Identification of the homolog of cell-counting factor in the cellular slime mold Dictyostelium discoideum. J. Biochem. Mol. Biol. Biophys. 6 351-355

Parkinson K, Buttery NJ, Wolf JB and Thompson CRL 2011 a simple mechanism for complex social behavior. PLoS Biol. 9 e1001039

Raper KB 1940 Pseudoplasmodium formation and organization in Dictyostelium discoideum. J. Elisha Mitchell Sci. Soc. 56 241-282

Raper KB 1984 The dictyostelids (Princeton: Princeton University Press)

Roisin-Bouffay C, Jang W, Caprette DR and Gomer RH 2000 A precise group size in Dictyostelium is generated by a cellcounting factor modulating cell-cell adhesion. Mol. Cell $6953-$ 959

Sathe S, Kaushik S, Lalremruata A, Aggarwal RK, Cavender JC and Nanjundiah V 2010 Genetic heterogeneity in wild isolates of cellular slime mold social groups. Microb. Ecol. 60 137-148

Stern C 1968 Genetic mosaics and other essays (Cambridge, MA: Harvard University Press)

Stern C and Tokunaga C 1967 Nonautonomy in differentiation of pattern-determining genes in Drosophila, I. The sex comb of eyeless-dominant. Proc. Natl. Acad. Sci. USA 57 658-664

Waddington $\mathrm{CH} 1942$ Canalization of development and the inheritance of acquired characters. Nature (London) 150 563-565

Watts DJ and Ashworth JM 1970 Growth of myxamoebae of the cellular slime mould Dictyostelium discoideum in axenic culture. Biochem. J. 119 171-174

ePublication: 08 July 2011 